\title{
Role of Interleukin-6 and Interleukin-8 Cytokines as Early Diagnostic Markers of Sepsis
}

\author{
Mohammad Moniruzzaman ${ }^{1}$, Ashesh Kumar Chowdhury ${ }^{2}$, ASM Areef Ahsan ${ }^{3}$, Md Zakiur \\ Rahman $^{4}$, Saimun Nahar Rumana ${ }^{5}$, Md. Abdullah Yusuf ${ }^{6}$, Gobindo Chandra Saha ${ }^{7}$
}

\begin{abstract}
${ }^{1}$ Assistant Professor, Department of Immunology, Bangladesh Institute of Health Science, Dhaka, Bangladesh; ${ }^{2}$ Associate Professor Department of Immunology, Bangladesh Institute of Research and Rehabilitation in Diabetes, Endocrine \& Metabolic Disorders, Dhaka, Bangladesh; ${ }^{3}$ Associate Professor, Department of Critical Care Medicine, Bangladesh Institute of Research and Rehabilitation in Diabetes, Endocrine and Metabolic Disorders, Dhaka, Bangladesh; ${ }^{4}$ Professor Department of Microbiology, Monno Medical College, Manikgonj, Bangladesh; ${ }^{5}$ Lecturer, Department of Physiology, Ibrahim Medical College, Dhaka, Bangladesh; ${ }^{6}$ Assistant Professor, Department of Microbiology, National Institute of Neurosciences \& Hospital, Dhaka, Bangladesh; ${ }^{7}$ Associate Professor, Department of Physiology, Cox’s Bazar medical College, Cox’s Bazar, Bangladesh
\end{abstract}

[Received: 1 July 2019; Accepted: 15 September 2019; Published: 1 December 2019]

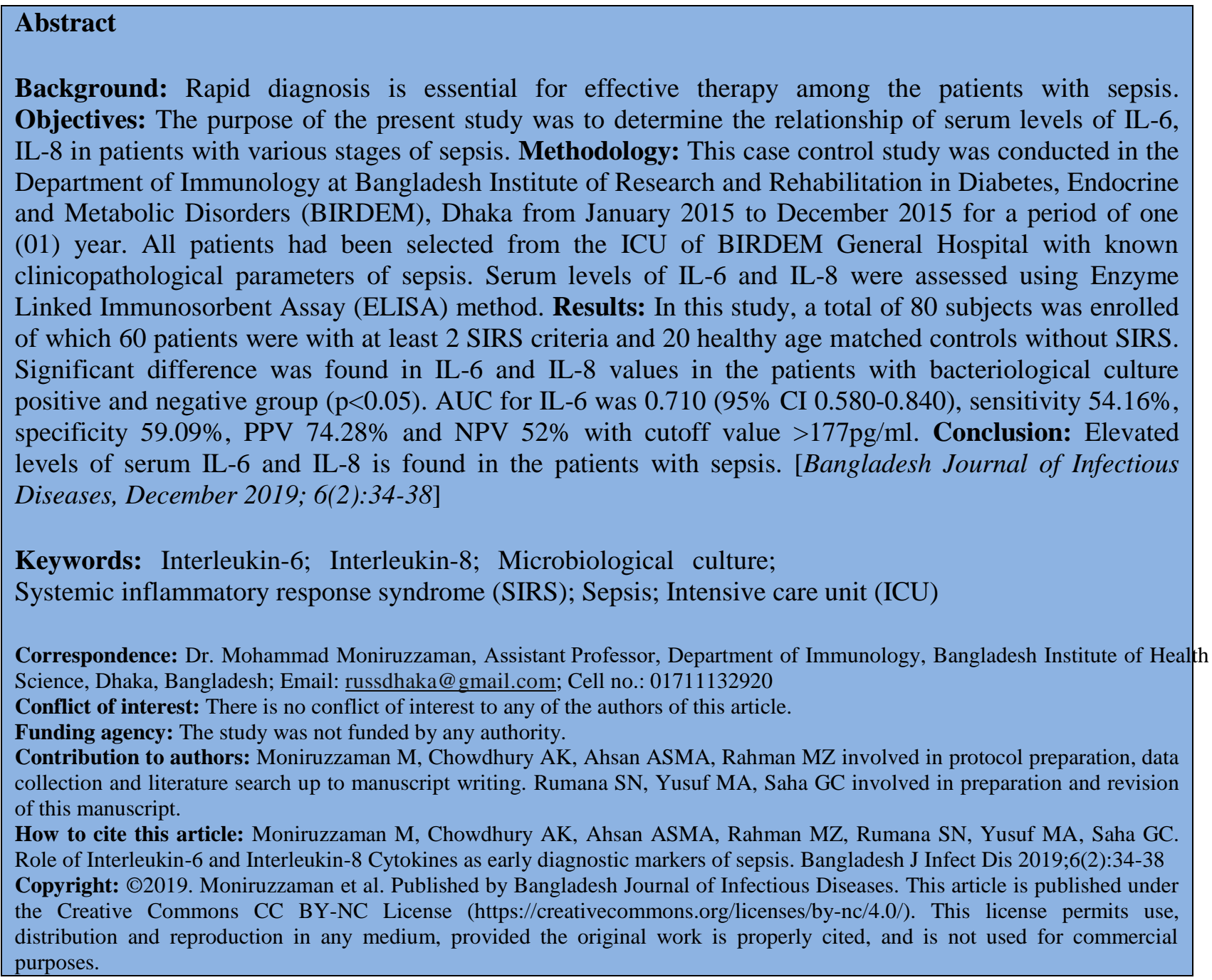




\section{Introduction}

The term 'sepsis' is used to define the systemic inflammatory response syndrome (SIRS) to an infectious agent. It is complicated to differentiate sepsis from other noninfectious conditions in critically ill patients admitted with systemic inflammatory response syndrome. Though we have new treatment modalities, advanced laboratory technique and clinical experience, mortality rates in sepsis are still higher ${ }^{1}$. Hospital mortality in ICU patients of Bangladesh suffering from severe sepsis was $49.2 \%$ which is very higher than other country $^{2}$. The critically ill patient frequently presents with similar clinical pictures in infection, SIRS, various severities of sepsis and organ dysfunction. This issue is of paramount importance given that therapies and outcomes greatly differ between patients with and those without sepsis. Thus, there is an unmet need for laboratory tools to determine SIRS and the various forms of sepsis early.

The incidence of sepsis and the number of sepsisrelated deaths are increasing day by day and among hospitalized patients by $8.7 \%$ per year ${ }^{3}$. Early diagnosis and appropriate therapy of sepsis is a daily challenge in intensive care units (ICU). An international prospective cohort study done among patients admitted to the 28 participating units in eight countries between May 1997 and May 1998 were followed until hospital discharge and overall 3034 infectious episodes (incidence: 21.1\%) were recorded at ICU admission ${ }^{4}$.

\section{Methodology:}

This case control study conducted at Department of Immunology, BIRDEM General Hospital, Dhaka in collaboration with Intensive Care Unit of BIRDEM General Hospital, Dhaka for a period of one year from 1st January 2015 to 31st December 2015. The study includes 80 subjects and purposive sampling done. Here 60 patients (Case) were chosen with at least 2 SIRS criteria and 20 age matched healthy control. All of them were above 18 years and after taking full medical history all of them examined properly and all medical data were reviewed. Selected patients under study were diagnosed as various stages of sepsis patient with different age group. Basic haematological, biochemical, microbiological laboratory data were recorded from laboratory report. IL-6 was measured by Enzyme linked Immunosorbent Assay (ELISA); IL-8 was measured by Sandwich Immunoassay. All patients were classified into SIRS, sepsis, severe sepsis, septic shock and MODS according to ACCP/SCCM definition which is now widely used. All data was checked and reviewed after collection. From the primary data obtained, tables and graph were made and interpreted. Levels of IL-6 and IL-8 were expressed as the means \pm SD or median \pm interquartile range. A p value of $<0.05$ was considered to be statistically significant. A nonparametric levene's test was used to verify the equality of variance in the samples data. The quantitative variables were analyzed by Mann Whitney $U$ test. Data was applied in the SPSS version 16 for statistical analysis. Their diagnostic utilities were compared using ROC curves.

\section{Results}

The study was performed in Intensive care unit (ICU), BIRDEM General Hospital, Dhaka. On admission all patients were examined and selected on the basis of SIRS criteria. All past and present data were reviewed carefully to conduct the study. A total of 80 subjects were include in the study, 60 of them were patients with at least 2 SIRS criteria and their mean age was $51.90 \pm 9.89$ years and 20 healthy controls with mean age of $49.65 \pm 9.58$ years. Among study population male were $51.2 \%$ (41) and female were $48.8 \%$ (39) in number. Commonest age range in patients was 51-60 years $(40 \%)$

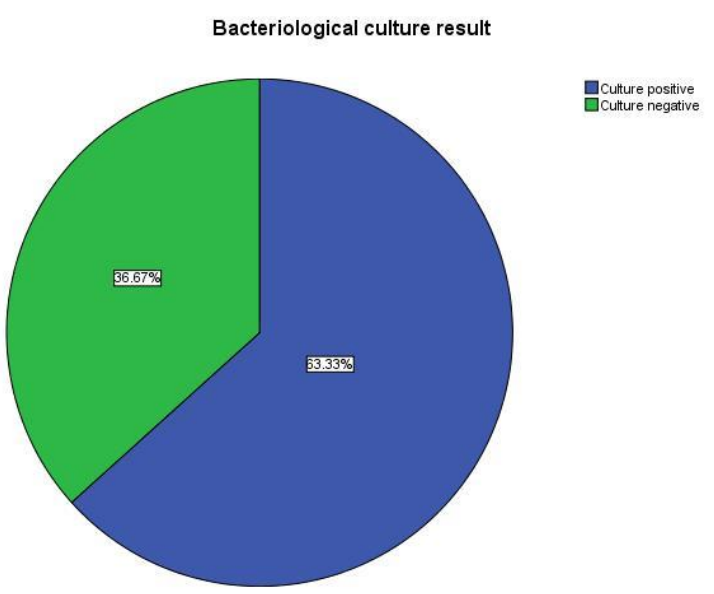

Figure I: Percentage of bacteriological culture result

All of the patients had at least 2 SIRS criteria and they were diagnosed clinically at various stages of sepsis. But SIRS criteria may appear in noninflammatory condition and without bacterial infection also. The gold standard method to prove sepsis is positive bacteriological culture. In this study $38(63.33 \%)$ patients were bacteriological 
culture positive and $22(36.67 \%)$ patients were bacteriological culture negative.

Staging of healthy individuals and patients according to clinicopathological parameter: In this study $20(25 \%)$ healthy individual were taken as control (No SIRS) and 60 (75\%) patients were categorized into SIRS, sepsis, severe sepsis, septic shock and MODS according to ACCP/SCCM guideline

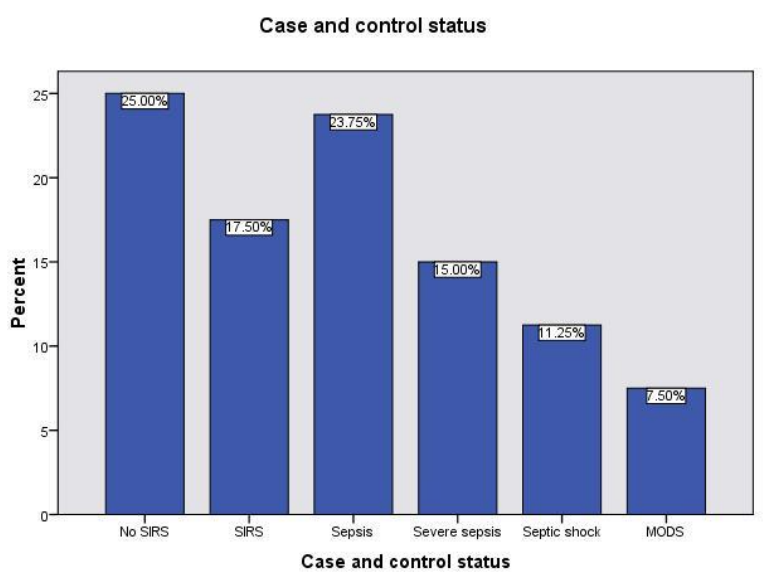

Figure II: Staging of sepsis

Distribution of organism pattern in sepsis: Among bacterial growth, the commonest organism was Klebsiella $(28.95 \%)$ followed by Escherichia coli (15.79\%), Acinetobacter (15.79\%), Pseudomonas aeruginosa (13.16\%), Enterococcus $(10.53 \%)$, Extended spectrum beta lactamases
(ESBL) (10.53\%) and Staphylococcus aureus $(5.26 \%)$.

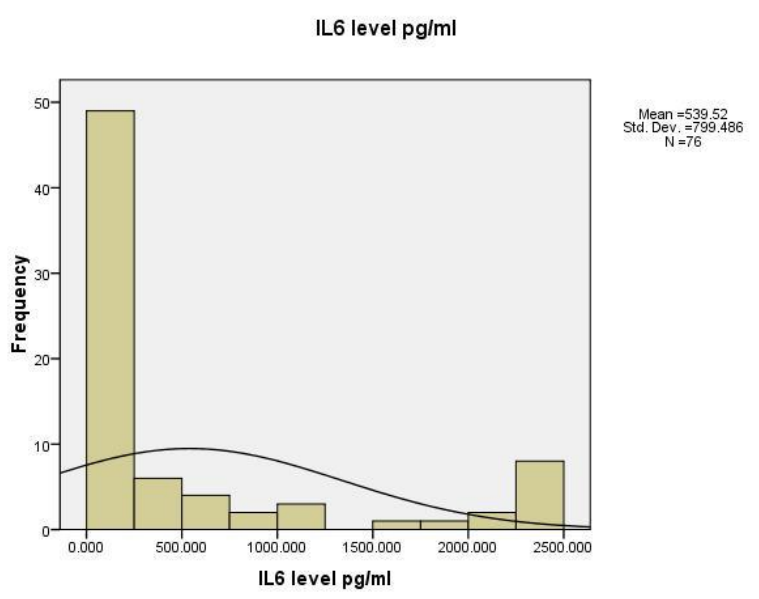

Figure III: Distribution of IL-6 data

The difference of IL-6 and IL-8 values between the patients and control: The sample data were not approximately normally distributed. A nonparametric levene's test was used to verify the equality of variance in the samples data $(\mathrm{p}<0.05)$ which confirmed the non-homogeneity of the distribution. Because of the nonhomogeneity, nonparametric Mann-Whitney $U$ test was used to see the difference between the groups. On MannWhitney $U$ test, statistically significant difference was found in between the IL- 6 and IL-8 values in the study subject and control population where $\mathrm{p}<0.05$ in all four biomarkers.

Table I: Significance of serum IL-6 and IL-8 values in patient and control.

\begin{tabular}{|l|l|c|c|c|}
\hline Biomarkers & Study group & Median & Interquartile range & P value \\
\hline \multirow{2}{*}{ IL6 level pg/ml } & Case & 202.55 & 957.93 & 0.000 \\
\cline { 2 - 4 } & Control & 4.43 & 8.03 & \\
\hline \multirow{2}{*}{ IL-8 level pg/ml } & Case & 29.31 & 168.10 & 0.002 \\
\cline { 2 - 4 } & Control & 16.34 & 15.39 & \\
\hline
\end{tabular}

The difference of IL-6 and IL-8 in between bacteriological culture positive and negative: The sample data were also not approximately normally distributed in bacteriological culture positive and negative patients. A nonparametric levene's test was used to verify the equality of variance in the samples data $(p<0.05)$ which confirmed the non-homogeneity of the distribution. Because of no homogeneity, nonparametric
Mann-Whitney $U$ test was used to see the difference between the groups. On MannWhitney U test, statistically significant difference was found in the IL-6, and IL-8 values in the bacteriological culture positive and negative group $(\mathrm{p}<0.05)$. 


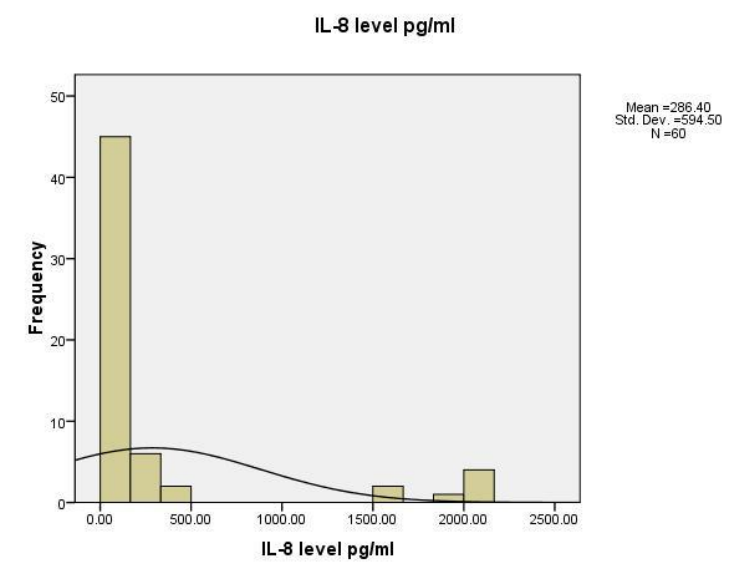

Figure IV: Distribution of IL-8 data

Table II: Diagnostic performance of IL-6, IL-8 to determine sepsis.

\begin{tabular}{|l|l|l|}
\hline Validity test & IL-6 & IL-8 \\
\hline Cutoff value & $177 \mathrm{pg} / \mathrm{ml}$ & $20 \mathrm{pg} / \mathrm{ml}$ \\
\hline Sensitivity & $54.16 \%$ & $76 \%$ \\
\hline Specificity & $59.09 \%$ & $50 \%$ \\
\hline PPV & $74.28 \%$ & $72.5 \%$ \\
\hline NPV & $52 \%$ & $55 \%$ \\
\hline Accuracy & $65 \%$ & $66.66 \%$ \\
\hline AUC & .710 & .681 \\
\hline
\end{tabular}

\section{Discussion}

In a cross-sectional point-prevalence study done over 454 ICUs in Germany in 2003 prevalence was $12.4 \%(95 \% \mathrm{CI}, 10.9-13.8 \%)$ for sepsis and $11.0 \%(95 \% \mathrm{CI}, \quad 9.7-12.2 \%)$ for severe sepsis including septic shock ${ }^{5}$. Despite the enormous investment in critical care resources, sepsis mortality ranges from $25 \%$ to $80 \%{ }^{6}$. Moreover, cases of severe sepsis are expected to rise for several reasons, including: increasing numbers of immunocompromised patients and aged population; wider use of invasive procedures; more resistant microorganisms. In point prevalence study $32.8 \%$ of 895 patients in ICUs had sepsis on a single day ${ }^{7}$. Physicians use various clinical and laboratory data to differentiate infectious from noninfectious conditions in newly admitted patients. But no single clinical or biological indicator of sepsis has gained unanimous acceptance.
Though blood culture is the gold standard and has higher sensitivity and specificity over the hematological value and cytokine but this highly sensitive microbiological parameter is not available in our community health situation and it is time consuming also. In these cases, several indicators have been proposed as new diagnostic tests to assess various stages of sepsis in critically ill patients.

Several attempts have been made to correlate cytokines levels with sepsis and patient prognosis. Among the cytokines, IL-6 and IL-8 are important mediator of host response to bacterial infection and has important role in inflammatory pathogenesis. These cytokines also stimulate release of acute-phase reactants such as CRP and PCT. These cytokines and acute phase proteins have been proposed to be the most promising candidates for diagnosis of sepsis in early stage. Few studies abroad showed combinatorial use of these markers as early diagnosis and prognosis of sepsis in ICU patients. As far best of our knowledge, no studies focusing on the significance of combinatorial use of these markers in sepsis patients of Bangladesh have been reported. In view of these conflicting findings and the utmost importance of the timely diagnosis of sepsis at time of admission in ICU, the present study was undertaken to prospectively investigate the diagnostic value of IL-6 and IL-8 in a group of severely ill patients admitted with sign of systemic inflammatory response syndrome in ICU.

\section{Conclusion}

Elevated levels of serum IL- 6 and IL- 8 is found in the patients with sepsis. These will help clinicians to make their decision in diagnosis of sepsis along with other systemic manifestation.

1. Døllner H, Vartten L, Austgulen R. Early diagbostic markers for neonatal sepsis: comparing C-reactive protein, Interleukin-6, Soluble tumor necrosis factor receptors and soluble adhesion molecules. J Clin Epiemiol 2001;54: 1251-7

2. Krueger M, Nauck MS, Sang S, et al. Cord blood levels of interleukin- 6 and interleukin- 8 for the immediate diagnosis of early onset infection in premature infants. Biol Neonate 2001;80:118-23

3. Martius JA, Roos T, Gora B, et al. Risk factors associated with early onset sepsis in premature infants. Obsetet Gynecol Reprod Biol 1999;85:151-8

4. Chiesa C, Pellegrini G, Panero A, et al. C-reactive protein interleukin-6 and procalcitonin in the immedial. Postnatal period and perinatal complications and infection. Clin Chem 2003;49:60-8

5. Chiesa C, Signore F, Assumma M. Serial measurement of C-reactive protein and interleukin-6 in the immediate postnatal period: Reference intervals and analysis of maternal and perinatal counfounders. Clin Chem 2001;47: 1016-22 
6. Shahkar L, Keshtkar A, Mirfazeli A, et al. The role of IL-6 for predicting neonatal sepsis: a systematic review and metaanalysis. Iran J Pediatr 2011;21:411-7

7. Heydarzadeh M, Movahedian AH, Mosayebi Z, et al. Predictive value of plasma interleukin-6 level in the diagnosis of early neonatal sepsis. KAUMS J 2012;16:229-34

8. Buck C, Bundschu J, Gallati H, et al. Interleukin-6: a sensitive parameter for the early diagnosis of neonatal bacterial infection. Pediatrics 1994;93:54-8

9. Wu Y, Wang M, Zhu Y, Lin S. Serum interleukin-6 in the diagnosis of bacterial infection in cirrhotic patients: a metaanalysis. Medicine (Baltimore) 2016;95:e5127

10. Lin S, Huang Z, Wang M, et al. Interleukin-6 as an early diagnostic marker for bacterial sepsis in patients with liver cirrhosis. J Crit Care 2015;30:732-8

11. Adib M, Navaei F, Bakhshiyani Z, et al. Evaluation of interleukin-6 in the early diagnosis of neonatal sepsis in compare with CRP. JIMS 2006;24:1-7
12. Kocabaş E, Sarikçioğlu A, Aksaray N, et al. Role of procalcitonin, C-reactive protein, interleukin-6, interleukin-8 and tumor necrosis factor-alpha in the diagnosis of neonatal sepsis. Turk J Pediatr 2007;49:7-20

13. Procianoy RS, Silveira RC. The role of sample collection timing on interleukin-6 levels in early-onset neonatal sepsis. J Pediatr 2004;80:407-10

14. Bender L, Thaarup J, Varming K, et al. Early and late markers for the detection of early-onset neonatal sepsis. Dan Med Bull 2008;55:219-23

15. Reyes CC, Munoz FG, Reyes D, et al. Role of cytokines (interleukin-1 $\beta, 6,8$, tumour necrosis factor-alpha, and soluble receptor of interleukin-2) and Creactive protein in the diagnosis of neonatal sepsis. Acta Paediatr 2003; 92:221

16. Kishimoto T. IL-6: from its discovery to clinical applications. Int Immunol 2010;22:347-52

17. Lin S, Huang Z, Wang M, et al. Interleukin-6 as an early diagnostic marker for bacterial sepsis in patients with liver cirrhosis. J Crit Care 2015; 30:732-8 